\title{
Caracterización de los ecosistemas costeros al norte del Área Protegida de Recursos Manejados Península de Guanahacabibes, Cuba
}

\author{
Characterization of coastal ecosystems north of the managed \\ resource protected area at the Guanahacabibes Peninsula, Cuba
}

\author{
Dorka Cobián ${ }^{1 *}$, Susana Perera², Alina Pérez², Susana Aguilar², Anmaris Álvarez", Zaimiuris \\ Hernández ${ }^{5}$, Leonardo Espinosa ${ }^{5}$, Héctor Salvat $^{6}$, Alain Alcalá ${ }^{7}$, Macario Ezquivel $^{7}$ y Leslie Hernández ${ }^{6}$
}

\begin{abstract}
RESUMEN
La Península de Guanahacabibes fue propuesta como Área Protegida de Recursos Manejados (APRM) en el Sistema Nacional de Áreas Protegidas de Cuba. Para validar esta propuesta se realizó un estudio de caracterización al área, en el marco del proyecto "Aplicación de un enfoque regional al manejo de las áreas protegidas marinas y costeras en los archipiélagos del sur de Cuba", con el objetivo de caracterizar los ecosistemas costeros y reafirmar que la ubicación y extensión diseñada del APRM responden a la protección de los principales valores naturales. Se evaluaron los manglares, las lagunas costeras y los pastos marinos, así como los sitios más importantes para las comunidades de aves marinas y acuáticas y las poblaciones de manatíes. Los muestreos se realizaron con metodologías validadas en el marco del proyecto. Se presentan los resultados, que constituyen la línea base del área, identificándose 149 especies de algas, 4 especies de fanerógamas marinas, 53 especies de peces del manglar y 39 especies de aves marinas y acuáticas. Los sitios seleccionados como prioridades de conservación y manejo de hábitats y especies incluyen los sitios de alimentación y nidificación de las aves marinas y acuáticas y la ubicación de dos zonas importantes para el monitoreo del manatí. Se identificaron las principales problemáticas del área, con el objetivo de realizar futuras acciones de conservación y manejo de los recursos naturales.
\end{abstract}

Palabras claves: Áreas protegidas, ecosistemas costeros, Guanahacabibes.

\begin{abstract}
The Guanahacabibes Peninsula was proposed as a Managed Resource Protected Area (APRM) in the Cuban National System of Protected Areas. In order to validate this proposal a study was conducted as part of the project "Implementation of a Regional Approach to the Management of Marine and Coastal Protected Areas in Southern Cuban Archipelagos", with the purpose of characterizing coastal ecosystems and reassuring that the APRM designed location and extension correspond to the protection of the main natural resources of the region. Mangroves, coastal lagoons and seagrass were assessed as well as important sites for seabird and waterfowl communities and manatee populations. Sampling was conducted with methods validated in the project. Results included the baseline of the area and identified 149 algae species, 4 seagrass species, 53 mangrove fish species, and 39 seabird and waterfowl species. The sites selected as priority for habitat and species conservation and management include feeding and nesting sites for seabirds and waterfowl and two important sites for the manatee. The main problems for the area were identified for future conservation and management actions.
\end{abstract}

Keywords: Protected areas, coastal ecosystems, Guanahacabibes.

1 Parque Nacional Guanahacabibes, Centro de Investigaciones y Servicios Ambientales (ECOVIDA), Ministerio de Ciencia, Tecnología y Medio Ambiente (CITMA). La Bajada, Pinar del Río, Cuba. dorkacobian@gmail.com*

2 Centro Nacional de Áreas Protegidas, CITMA.

3 Centro de Investigaciones y Servicios Ambientales (ECOVIDA), CITMA.

4 Centro de Investigaciones Marinas, Universidad de La Habana.

5 Parque Nacional Cayos de San Felipe.

6 Centro de Investigaciones de Ecosistemas Costeros Cayo Coco, CITMA.

7 Instituto de Oceanología, CITMA.

Recibido 28 XI 2012

Aceptado 11 VII 2013

DOI: http://dx.doi.org/10.15359/revmar.5.3 


\section{INTRODUCCIÓN}

Los humedales son considerados ecosistemas muy importantes, debido a la gran biodiversidad que albergan. Además, resultan de mucho beneficio porque intervienen en otros procesos tales como, la estabilización de costas, la depuración de aguas, el control de inundaciones, entre otros. Sin embargo, conjuntamente con los bosques lluviosos, los humedales se encuentran entre los ecosistemas más amenazados del mundo, debido a su transformación por acciones antrópicas y a la contaminación (Ramsar, 2013).

En Cuba, estos ecosistemas se destacan por su extensión, productividad y riqueza. La Península de Guanahacabibes se caracteriza por la presencia de importantes manglares, lagunas costeras, ciénagas, pastos marinos, así como arrecifes coralinos en muy buen estado de conservación.

La mayoría de los estudios en la zona costera de la península se han realizado en la costa sur, destacándose entre ellos, la sistemática de algas (Valdivia et al. 2004), de moluscos marinos (Espinosa et al. 2005), ecología de corales (Valdivia et al. 2004; González-Ferrer et al. 2007; Caballero et al. 2007; Perera, 2010), de moluscos fluviales (Vázquez \& Cobián, 2008), de peces (Claro \& Cantelar, 2003; Cobián \& Chevalier Monteagudo, 2009; Cobián et al. 2011), iguanas (Cobián et al. 2008) y de la anidación de tortugas marinas (Azanza et al. 2003; 2012).

En la costa norte solo se han realizado estudios de sistemática de moluscos marinos en zonas muy puntuales (Espi- nosa et al. 2005; 2007), existiendo vacíos de información sobre la diversidad marina en los manglares, las lagunas costeras y los pastos marinos, desconociéndose su estado actual de salud y conservación.

En la zona más interna de la península se localizan cinco asentamientos rurales con una población total de 1719 habitantes y hacia los límites se registra un total de 4217 habitantes, principalmente en las comunidades de $\mathrm{La} \mathrm{Fe}$, Manuel Lazo y La Güira, siendo la comunidad costera de La Fe la de mayor incidencia sobre los recursos marinos de la costa norte del Área Protegida de Recursos Manejados (APRM).

En el marco del proyecto "Aplicación de un enfoque regional al manejo de las áreas protegidas marinas y costeras en los archipiélagos del sur de Cuba" que ejecuta el Centro Nacional de Áreas Protegidas, se realizó una expedición, con los objetivos de caracterizar los ecosistemas marinos y costeros de la costa norte de la península, de comprobar que la extensión de los límites diseñados del área protegida incluían todos los recursos marinos, además de establecer la línea base de la zona, e identificar los posibles hábitats para la población de manatíes (Trichechus manatus manatus) y los sitios de alimentación y nidificación para las aves marinas y acuáticas.

\section{MATERIALES Y MÉTODOS}

\section{Área de estudio}

La zona de estudio comprendió el litoral norte de la Península de Guanahacabibes, la cual se extiende desde el 
Cabo de San Antonio (21 $1^{\circ}$ 52' 02.9" $\mathrm{N}$; $84^{\circ} 57^{\prime} 16.4^{\prime \prime} \mathrm{W}$ ), incluyendo los Cayos de la Leña $\left(21^{\circ} 55^{\prime} 04.0^{\prime \prime} \mathrm{N}\right.$; $84^{\circ} 48^{\prime} 38.0^{\prime \prime} \mathrm{W}$ ), hasta el interior de la Bahía de la Fe $\left(22^{\circ} 01\right.$ ' 27.7" N; $\left.084^{\circ} 18^{\prime} 97.5^{\prime} \mathrm{W}\right)$, ocupando aproximadamente $100 \mathrm{~km}$ de extensión. Esta área está cubierta en su totalidad por bosques de mangle, lagunas costeras y canales, predominando hacia la zona marina la especie Rhizophora mangle (mangle rojo). Le siguen a esta especie los pastos marinos hasta la isobata de $3 \mathrm{~m}$. El área se dividió en cinco zonas: Cabo de San Antonio, Cayos de la Leña, Palma Sola-Carabelita, Punta PlumajeMelones y Bahía de la Fe (Fig. 1), en las cuales se realizaron 20 estaciones distribuidas por toda la costa norte (Cuadro 1, Fig. 2), que se caracterizaron dependiendo del tipo de ecosistema y de la existencia de las especies a estudiar.

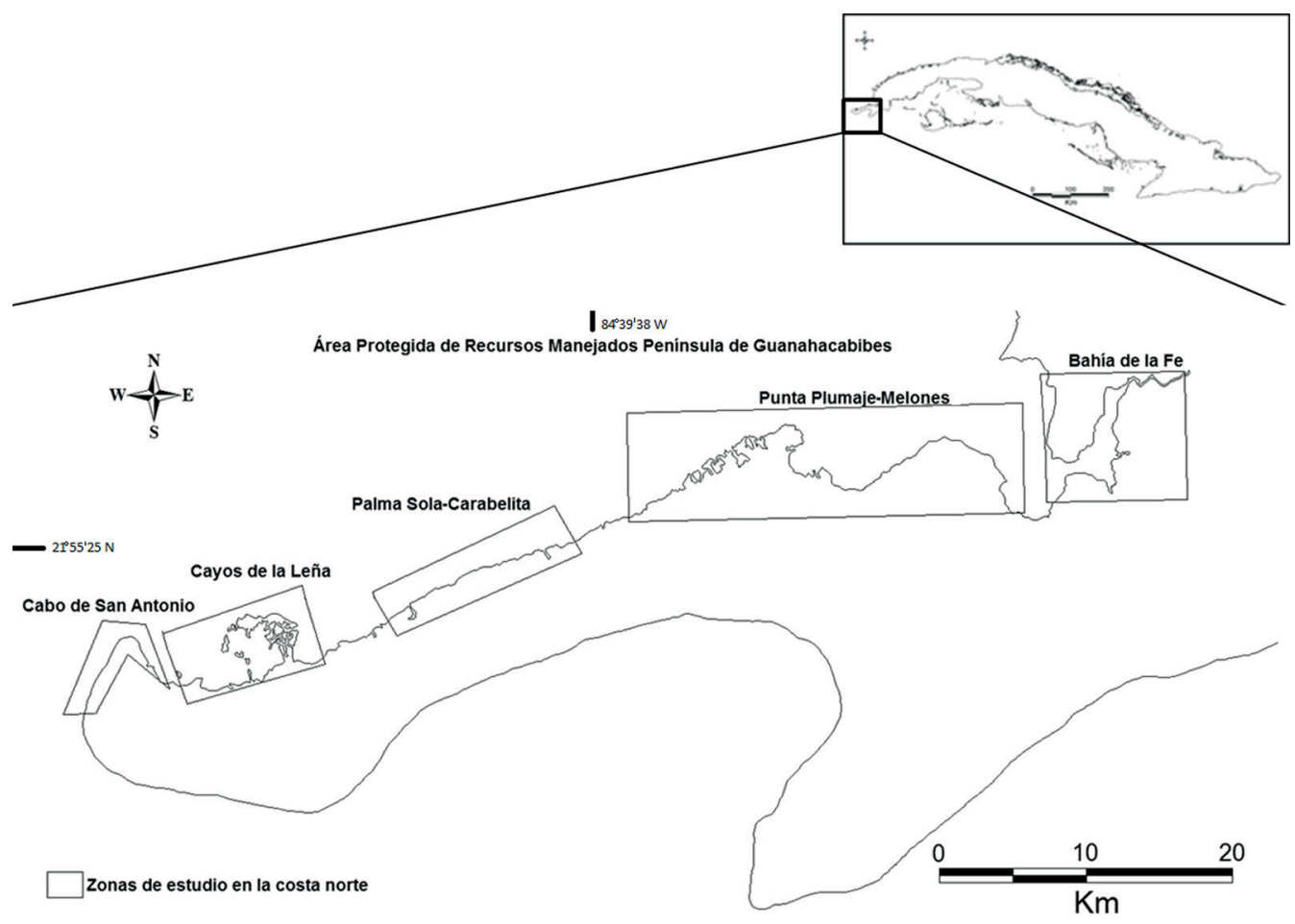

Fig. 1. Ubicación de las zonas estudiadas en el APRM Península de Guanahacabibes Fig. 1. Location of the studied sites in the Guanahacabibes Peninsula APRM

\section{Metodologías}

\section{Descripción de áreas de estudio}

Cabo de San Antonio: Se extiende desde el Cabo de San Antonio hasta Los Morros. Esta zona cuenta con playas con fondos arenosos, con pastos marinos formados por las especies Thalassia testudinum y Syringodium filiforme, con un $69 \%$ y $34 \%$ de cobertura, respectivamente. Hacia el norte le siguen lagunas 
Cuadro 1. Descripción de los parámetros abióticos en las estaciones muestreadas. Temperatura de fondo (Tf), temperatura de superficie (Ts), salinidad de fondo (Sf), salinidad de superficie (Ss), profundidad (P), visibilidad (V) y velocidad del viento (V.V)

Table 1. Description of abiotic parameters in studied sites. Bottom temperature (Tf), surface temperature (Ts), bottom salinity (Sf), surface salinity (Ss), depth (P), visibility (V), and wind speed (V.V)

\begin{tabular}{|c|c|c|c|c|c|c|c|c|c|}
\hline Zonas & Coordenadas & $\operatorname{Tf}\left({ }^{\circ} \mathrm{C}\right)$ & $\begin{array}{c}\text { Ts } \\
\left({ }^{\circ} \mathrm{C}\right)\end{array}$ & Sf (UPS) & Ss (UPS) & $\mathbf{P}(\mathbf{m})$ & $V(\%)$ & V.V & Sustrato \\
\hline \multicolumn{10}{|c|}{ Cabo de San Antonio } \\
\hline Faro Roncali & $\begin{array}{l}21^{\circ} 52^{\prime} 02.9^{\prime \prime} \mathrm{N} \\
84^{\circ} 57^{\prime} 16.4^{\prime \prime} \mathrm{W}\end{array}$ & 27.4 & 27.4 & 34.6 & 34.6 & 2.2 & 100 & 3 & $\begin{array}{l}\text { arenoso y } \\
\text { rocoso }\end{array}$ \\
\hline Tumbas & $\begin{array}{l}21^{\circ} 53^{\prime} 58.1^{\prime \prime} \mathrm{N} \\
84^{\circ} 56^{\prime} 19.7^{\prime \prime} \mathrm{W}\end{array}$ & 26 & 26 & 34.8 & 34.8 & 3.4 & 100 & 4 & $\begin{array}{l}\text { arena y } \\
\text { piedra }\end{array}$ \\
\hline Los Morros & $\begin{array}{l}21^{\circ} 54^{\prime} 04.7^{\prime \prime} \mathrm{N} \\
84^{\circ} 54^{\prime} 26.2^{\prime \prime} \mathrm{W}\end{array}$ & 26 & 26 & 34.8 & 34.8 & 2.7 & 100 & 1 & $\begin{array}{l}\text { piedra con } \\
\text { turba }\end{array}$ \\
\hline \multicolumn{10}{|c|}{ Cayos de la Leña } \\
\hline Cayo Triste & $\begin{array}{l}21^{\circ} 53^{\prime} 49.3^{\prime \prime} \mathrm{N} \\
84^{\circ} 53^{\prime} 12.2^{\prime \prime} \mathrm{W}\end{array}$ & 25.6 & 25.6 & 35 & 35 & 1.9 & 100 & 2 & arenoso \\
\hline $\begin{array}{l}\text { Cayos de la } \\
\text { Leña } 1\end{array}$ & $\begin{array}{l}21^{\circ} 55^{\prime} 04.0 ” \mathrm{~N} \\
84^{\circ} 48^{\prime} 38.0^{\prime \prime} \mathrm{W}\end{array}$ & 24.7 & 24.6 & 34.2 & 34.2 & 2.5 & 100 & 2 & arenoso \\
\hline $\begin{array}{l}\text { Cayos de la } \\
\text { Leña } 2\end{array}$ & $\begin{array}{l}21^{\circ} 55^{\prime} 16.5^{\prime \prime} \mathrm{N} \\
84^{\circ} 49^{\prime} 11.8^{\prime \prime} \mathrm{W}\end{array}$ & 24.6 & 24.8 & 35 & 35 & 5.2 & 100 & 2 & fango claro \\
\hline $\begin{array}{l}\text { Cayos de la } \\
\text { Leña } 3\end{array}$ & $\begin{array}{l}21^{\circ} 55^{\prime} 19.3 ” \mathrm{~N} \\
84^{\circ} 51^{\prime} 27.5^{\prime \prime} \mathrm{W}\end{array}$ & 26 & 26 & 35.1 & 35.1 & 2 & 100 & 1 & arenoso \\
\hline $\begin{array}{l}\text { Cayos de la } \\
\text { Leña } 4\end{array}$ & $\begin{array}{l}21^{\circ} 55^{\prime} 36.2^{\prime \prime} \mathrm{N} \\
84^{\circ} 49^{\prime} 54.6 ” \mathrm{~W}\end{array}$ & 25.6 & 25.7 & 35.4 & 35.3 & 1.7 & 100 & 2 & fango claro \\
\hline $\begin{array}{l}\text { Cayos de la } \\
\text { Leña } 5\end{array}$ & $\begin{array}{l}21^{\circ} 55^{\prime} 29.4^{\prime \prime} \mathrm{N} \\
84^{\circ} 49^{\prime} 45.9^{\prime \prime} \mathrm{W}\end{array}$ & 26 & 26 & 35 & 35 & 2 & 100 & 3 & fango claro \\
\hline $\begin{array}{l}\text { Cayos de la } \\
\text { Leña } 6\end{array}$ & $\begin{array}{l}21^{\circ} 55^{\prime} 18.4^{\prime \prime} \mathrm{N} \\
84^{\circ} 49^{\prime} 35.0^{\prime \prime} \mathrm{W}\end{array}$ & 26 & 26 & 35 & 35 & 3 & 100 & 3 & fango claro \\
\hline $\begin{array}{l}\text { Cayos de la } \\
\text { Leña } 7\end{array}$ & $\begin{array}{l}21^{\circ} 54^{\prime} 26.5^{\prime \prime} \mathrm{N} \\
84^{\circ} 49^{\prime} 16.5^{\prime \prime} \mathrm{W}\end{array}$ & 26 & 26 & 35.3 & 35.3 & 2.3 & 100 & 3 & fango \\
\hline $\begin{array}{l}\text { Cayos de la } \\
\text { Leña } 8\end{array}$ & $\begin{array}{l}21^{\circ} 54^{\prime} 27.5^{\prime \prime} \mathrm{N} \\
84^{\circ} 51^{\prime} 20.5^{\prime \prime} \mathrm{W}\end{array}$ & 24.8 & 24.8 & 35.2 & 35.2 & 1.7 & 100 & 2 & fango \\
\hline $\begin{array}{l}\text { Cayos de la } \\
\text { Leña } 9\end{array}$ & $\begin{array}{l}21^{\circ} 54^{\prime} 12.8^{\prime \prime} \mathrm{N} \\
84^{\circ} 50^{\prime} 47.4^{\prime \prime} \mathrm{W}\end{array}$ & 24.7 & 24.8 & 35.3 & 35.4 & 3.5 & 100 & 2 & fango claro \\
\hline $\begin{array}{l}\text { Cayos de la } \\
\text { Leña } 10\end{array}$ & $\begin{array}{l}21^{\circ} 53^{\prime} 24.1^{\prime \prime} \mathrm{N} \\
84^{\circ} 50^{\prime} 28.1^{\prime \prime} \mathrm{W}\end{array}$ & 25,3 & 25,3 & 34,5 & 34,5 & 1 & 100 & 2 & fango y rocas \\
\hline \multicolumn{10}{|c|}{ Palma Sola-Carabelita } \\
\hline Carabelita & $\begin{array}{l}21^{\circ} 56^{\prime} 8.24^{\prime \prime} \mathrm{N} \\
84^{\circ} 44^{\prime} 20.21^{\prime \prime} \mathrm{W}\end{array}$ & 25.6 & 25.8 & 33.5 & 34.6 & 1 & 100 & 3 & arenoso \\
\hline Palma Sola & $\begin{array}{l}21^{\circ} 58^{\prime} 12.21^{\prime \prime} \mathrm{N} \\
84^{\circ} 39^{\prime} 2.29 ” \mathrm{~W}\end{array}$ & 26 & 27 & 30.5 & 35.1 & 2.3 & 100 & 2 & fango claro \\
\hline \multicolumn{10}{|c|}{ Punta Plumaje-Melones } \\
\hline Punta Plumaje & $\begin{array}{l}22^{\circ} 02^{\prime} 22.0^{\prime \prime} \mathrm{N} \\
84^{\circ} 28^{\prime} 47.2^{\prime \prime} \mathrm{W}\end{array}$ & 25.6 & 25.6 & 34.6 & 34.6 & 3.4 & 100 & 2 & arenoso \\
\hline $\begin{array}{l}\text { Ensenada de } \\
\text { Melones }\end{array}$ & $\begin{array}{l}22^{\circ} 02^{\prime} 03.1 ” \mathrm{~N} \\
84^{\circ} 24^{\prime} 12.8^{\prime \prime} \mathrm{W}\end{array}$ & 25.2 & 25.2 & 34.3 & 34.3 & 2.1 & 100 & 2 & arenoso \\
\hline \multicolumn{10}{|c|}{ Bahía de la Fe } \\
\hline $\begin{array}{l}\text { Bahía de la } \\
\text { Fe } 1\end{array}$ & $\begin{array}{l}22^{\circ} 01^{\prime} 27.7^{\prime \prime} \mathrm{N} \\
84^{\circ} 18^{\prime} 97.5^{\prime \prime} \mathrm{W}\end{array}$ & 25.3 & 25.3 & 33.8 & 33.8 & 1.4 & 100 & 3 & fango claro \\
\hline $\mathrm{La} \mathrm{Fe} 2$ & $\begin{array}{l}22^{\circ} 03^{\prime} 26.4^{\prime \prime} \mathrm{N} \\
84^{\circ} 16^{\prime} 36.6^{\prime \prime} \mathrm{W}\end{array}$ & 25.1 & 25.2 & 33.8 & 33.7 & 2.1 & 100 & 3 & fango \\
\hline $\begin{array}{l}\text { Río de } \\
\text { Guadiana } 3\end{array}$ & $\begin{array}{l}22^{\circ} 04^{\prime}, 23.3^{\prime \prime} \mathrm{N} \\
84^{\circ} 15^{\prime} 39.6^{\prime \prime} \mathrm{W}\end{array}$ & 25.5 & 25.8 & 33.5 & 32.6 & 3.2 & 100 & 3 & fango \\
\hline $\begin{array}{l}\text { Río de } \\
\text { Guadiana } 4\end{array}$ & $\begin{array}{l}22^{\circ} 04^{\prime} 15.7^{\prime \prime} \mathrm{N} \\
84^{\circ} 14^{\prime} 43.2^{\prime \prime} \mathrm{W}\end{array}$ & 25.9 & 25.7 & 32.9 & 32.6 & 4.1 & 100 & 2 & fango \\
\hline
\end{tabular}




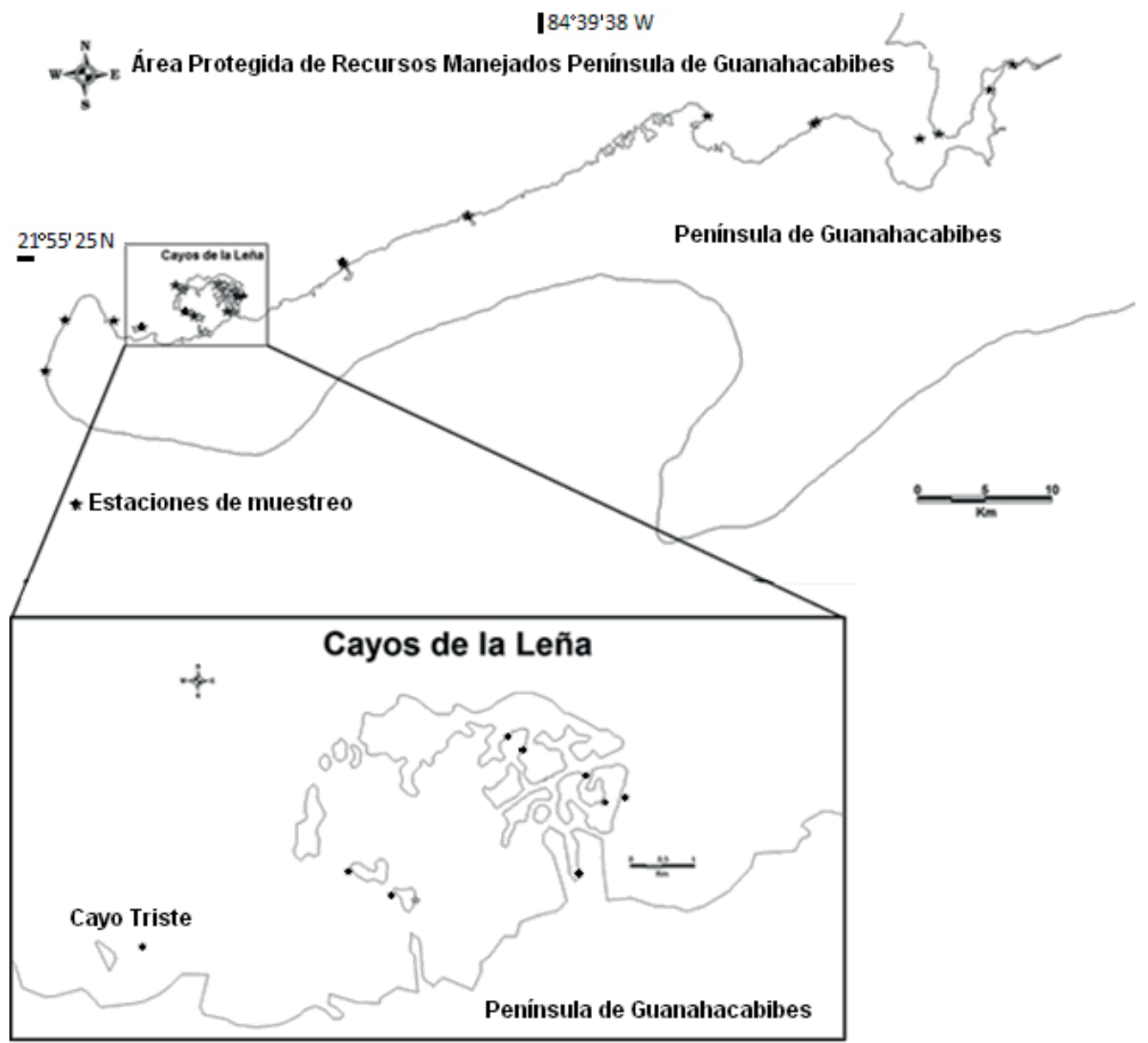

Fig. 2. Ubicación de las 20 estaciones de muestreo en el APRM Península de Guanahacabibes Fig. 2. Location of the 20 survey areas in the Guanahacabibes Peninsula APRM

interiores y manglares achaparrados, en estos últimos se observan daños ocasionados por el huracán Iván en el 2004, sin embargo, se observa regeneración natural de $R$. mangle.

Cayos de la Leña: Cayos formados por mangle rojo, cuya altura varía de 1.5 a $4 \mathrm{~m}$. Se encuentran formando una amplia red de canales y en su interior se localiza la Laguna de Bolondrón. De forma general, los manglares están en buen estado sin ningún disturbio antrópico, aunque se observaron pequeñas áreas afectadas en fase de recuperación. Su sistema de canales y lagunas de profundidades variables lo convierten en un importante sitio de crianza de juveniles de invertebrados y peces comerciales, además de refugio para el manatí y las aves acuáticas y marinas.

Palma Sola-Carabelita: No existe un sistema de canales bien definido. Esta zona se encuentra más afectada por los vientos invernales (nortes), por lo que durante la época de invierno existe un gran oleaje en la zona, lo cual provoca suspensión de sedimentos, lo 
que hace a esta zona más inestable y menos favorable para el asentamiento de los organismos sésiles en sus raíces y como refugio para los peces. En Palma Sola se encuentra una laguna de bolsa muy resguardada, que constituye un importante refugio para los manatíes y las aves marinas y acuáticas.

Punta Plumaje-Melones: Los manglares más representativos de tierra se encuentran en Punta Plumaje. En esta zona existe un sistema de canales y lagunas interiores que se comunican entre sí y permiten la existencia de grandes extensiones de bosque. Se encontraron los individuos más desarrollados y saludables con una altura estimada de $6 \mathrm{~m}$. A este ecosistema le siguen las marismas, sitios importantes para la alimentación de las aves acuáticas y marinas. El área se encuentra muy conservada y protegida de los vientos invernales (nortes), identificándose un sitio de nidificación de aves marinas, específicamente de cocos. En la zona intermareal, a poca profundidad, las raíces del mangle rojo forman un entramado que constituye un refugio ideal para peces e invertebrados comerciales. Los hábitats existentes en esta zona presentaron un elevado estado de conservación, siendo esta zona de vital importancia para las especies costeras y marinas.

Bahía de la Fe: Esta zona se encuentra muy resguardada por la estructura de la Ensenada de la Fe, en la que desemboca el Río Guadiana, cuyo aporte de fuentes de agua dulce es muy importante. Esta área se caracteriza por presentar riveras pobladas por mangle rojo, y en la parte sumergida, abundancia de pastos marinos. La composición y estructura de la zona favorece la estabilidad de las comunidades de aves acuáticas y marinas, proporcionándoles refugio y alimento, de igual manera para las poblaciones de manatíes. Sin embargo, se detectó contaminación en la desembocadura del Río Guadiana, que podria ser producto de desechos por aguas albañales y por vertimiento de industrias, pero en la actualidad no se conoce el nivel de polución ni el grado de afectación a la integridad de los ecosistemas y especies de la zona.

Durante el mes de noviembre del 2010 y septiembre del 2011 se realizaron campañas de muestreo durante diez días, en las que se ejecutaron 23 estaciones (14 para algas y pastos marinos y 9 manglares) de muestreo distribuidas en las cinco zonas, en las cuales se registraron los siguientes parámetros abióticos: temperatura del agua (superficie y fondo) y salinidad (superficie y fondo), medida con ecosonda $\left( \pm 0.2^{\circ} \mathrm{C}\right.$ y $\left.\pm 0.2 \mathrm{PSU}\right)$. Se tomaron las condiciones del mar a través de la Escala Beaufort adaptada a partir de la lectura de la velocidad del viento, la visibilidad vertical mediante el uso del disco Sechii, así como la profundidad y el tipo de sustrato. En cada zona se tuvieron en cuenta la presencia de amenazas como: erosión, turbidez, contaminación y desechos sólidos.

La clasificación de las algas se realizó in situ, excepto aquellas que no pudieran identificarse, en cuyo caso el material se recolectó y se fijó al 5\% en formalina. Posteriormente fueron cla- 
sificadas en el laboratorio. Para la determinación de las especies de algas se tomaron como referencias las obras de Taylor (1960) y Littler \& Litter (2000).

En cada estación de pastos marinos se ubicaron tres transeptos paralelos a la costa, aproximadamente al inicio, centro y final. En cada transepto se ubicaron al azar 12 cuadrados de $25 \times 25 \mathrm{~cm}$, para un total de 36 cuadrados. En cada uno se contaron los vástagos por especie de angiosperma marina, la altura de la canopia, el grado de epifitismo, y la cobertura de otras especies asociadas al pasto en el bentos (esponjas, gorgonias, anémonas, etc.) o macroalgas por grupos morfofuncionales. La metodología aplicada es un compendio de las principales variables de diferentes métodos de monitoreo de pastos marinos (CARICOMP, 2001), Seagrass Watch (McKenzie et al. 2003) y SeagrassNet (Short \& Wyllie-Echeverría, 1996).

En los manglares se ubicaron 9 estaciones para analizar las asociaciones de peces, se muestrearon 10 transectos lineales de $30 \mathrm{~m}$ de largo por 2 de ancho (Brock, 1954; Rogers et al. 1994), ocupando un área total de $600 \mathrm{~m}^{2}$. La profundidad varió desde $50 \mathrm{~cm}$ hasta 3 $\mathrm{m}$. Se registraron todas las especies, el número de individuos por especie y la talla estimada de cada pez.

Para conocer la composición de los organismos sésiles de las raíces sumergidas de los manglares se realizó una caracterización en seis estaciones en tres de las zonas visitadas. Para ello, a cada raíz se le determinó el tamaño (cm) y se le midió la cobertura de los organismos sésiles $(\mathrm{cm})$, en la zona que se consideró como el frente de la raíz (L. Hernández, comunicación personal, noviembre 20, 2011).

El censo de la población de manatíes se realizó a través de recorridos diarios que cubrían toda el área de estudio; en esta se efectuó una búsqueda activa de los animales a una velocidad de entre tres y cuatro nudos de la embarcación (Smethurst \& Nietschmann, 1999). En el estudio participaron cuatro observadores. También se realizaron ocho estaciones fijas desde la embarcación, en este método se abarcaron $360^{\circ}$ del área durante un tiempo de 30 minutos, para localizar a los animales que pudiesen estar sumergidos.

Para el inventario de la comunidad de aves marinas y acuáticas primeramente se seleccionaron los sitios más probables como hábitat para el refugio y la alimentación de aves acuáticas, utilizando imágenes de satélite de la costa norte de Guanahacabibes. En los sitios seleccionados de las cinco zonas trabajadas, se utilizaron dos métodos para el conteo: Itinerarios de censo y Conteos en punto fijo. En los Itinerarios de censo se registraron todos los individuos sin prefijar un ancho a cada lado, tratando de no contar las mismas aves en varias oportunidades. Estos se realizaron a pie, en bote por los canales bordeados de manglar y durante el recorrido del barco. Los Conteos en punto fijo se utilizaron principalmente en lagunas costeras, donde registramos todos los individuos de cada especie vistos u oídos en un período de tiempo determinado (30 minutos) sin prefijar un radio. El análisis de las colonias de 
nidificación incluyó: estimado de la colonia, tipo de hábitat, sustrato de nidificación, área que ocupa la nidificación y desarrollo de la colonia (construcción del nido, huevos, huevos más pichones, pichones más volantones y solo volantones) (L. Mujica, comunicación personal, noviembre 15, 2011).

\section{RESULTADOS Y DISCUSIÓN}

\section{Estructura biológica}

En el área se identificaron 149 especies de macroalgas, de las cuales, 55 especies son del Phylum Rhodophyta, 12 especies del Phylum Ochrophyta y 82 especies del Phylum Chlorophyta. La estación con mayor diversidad de algas fue la cercana a Cayo Triste, con 39 especies, coincidiendo con los mayores registros de abundancias de Halodule wrightii y T. testudinum. En otras estaciones donde el pasto marino fue poco o medianamente denso también se identificaron totales de 30 especies de algas.

Los pastos marinos están formados principalmente por las fanerógamas T. testudinum, S. filiforme, $H$. wrigthii y Halophila engelmanni. Se observaron praderas monoespecíficas de T. testudinum y $H$. wrigthii y mixtas formadas por T. testudinum, S. filiforme y H. wrigthii. Estos pastos marinos se desarrollan a partir de las zonas cercanas a las raíces de los mangles hasta las profundidades entre 2 y $3 \mathrm{~m}$, en diversos tipos de fondos. Los sustratos más comunes fueron: arenosos, fangosos, areno-fangoso y rocosos con deposición de arena y fango.

Las fanerógamas presentaron den- sidades medianas y altas, ocupando en algunos sitios el $100 \%$ de la cobertura del fondo. Con estas características se destacan los Cayos de la Leña, que presentaron pastos marinos formados por T. testudinum, S. filiforme y H. wrigthii, que cubren completamente el fondo y que presentan muy altas alturas de canopia de T. testudinum $(75-80 \mathrm{~cm})$. También presentaron estas características los sitios de la Bahía de la Fe, donde las praderas de T. testudinum cubren todo el fondo (Cuadro 2). El predominio de T. testudinum refleja una condición estable en el ecosistema de pastos marinos (Martínez et al. 2011).

De forma general, las praderas presentan un elevado estado de salud y una gran diversidad de organismos. Entre los principales grupos se encuentran: macroalgas, moluscos, equinodermos, esponjas, crustáceos, corales y tunicados. Entre las macroalgas se destacan los grupos de las calcáreas y carnosas, siendo Halimeda sp. y Dictyota sp. los géneros más abundantes. De las especies de invertebrados, Oreaster reticulatus fue la más abundante, lo cual reafirma el grado de conservación de estos ecosistemas y el bajo impacto humano, esto coincide con los resultados encontrados por Metaxas et al. (2002) en mares tropicales. La costa norte de la península está actuando como un reservorio de esta especie, que en la actualidad sus poblaciones han disminuido significativamente y son consideradas extintas en zonas de gran desarrollo costero (Myers \& Ottensmeyer, 2005).

Mantener el estado actual de conservación de los pastos marinos en el 
Cuadro 2. Porcentajes de cobertura (\% Cober), número de vástagos ( $\mathrm{N}^{\circ}$ Vást) y alturas de canopia (Alt can $(\mathrm{cm})$ de las fanerógamas Thalassia testudinum, Syringodium filiforme y Halodule wrightii y porcentaje de cobertura de macroalgas (\% Mac)

Table 2. Percent cover (\% Cober), number of shoots $\left(\mathrm{N}^{\circ}\right.$ Vast), and canopy height (Alt can $(\mathrm{cm})$ ) of Thalassia testudinum, Syringodium filiforme and Halodule wrightii phanerogams, and percent cover of macroalgae (\% Mac)

\begin{tabular}{|c|c|c|c|c|c|c|c|c|c|c|}
\hline \multirow[b]{2}{*}{ Estaciones } & \multicolumn{3}{|c|}{ Thalassia testudinum } & \multicolumn{3}{|c|}{ Syringodium filiforme } & \multicolumn{3}{|c|}{ Halodule wrightii } & \multirow{2}{*}{$\begin{array}{c}\% \\
\text { Мac }\end{array}$} \\
\hline & $\begin{array}{c}\% \\
\text { Cober }\end{array}$ & $\begin{array}{c}\mathbf{N}^{\mathbf{o}} \\
\text { Vást }\end{array}$ & $\begin{array}{c}\text { Alt can } \\
\text { (cm) }\end{array}$ & $\begin{array}{c}\% \\
\text { Cober }\end{array}$ & $\begin{array}{c}\mathbf{N}^{0} \\
\text { Vást }\end{array}$ & $\begin{array}{c}\text { Alt can } \\
\text { (cm) }\end{array}$ & $\begin{array}{c}\% \\
\text { Cober }\end{array}$ & $\begin{array}{c}\mathbf{N}^{\mathbf{o}} \\
\text { Vást }\end{array}$ & $\begin{array}{c}\text { Alt can } \\
(\mathrm{cm})\end{array}$ & \\
\hline \multicolumn{11}{|c|}{ Cabo de San Antonio } \\
\hline Faro Roncali & 69 & 39 & 15 & 39 & 5 & 15 & 0 & 0 & 0 & 4 \\
\hline Los Morros & 64 & 23 & 22 & 0 & 0 & 0 & 0 & 0 & 0 & 24 \\
\hline Manglares de tierra 1 & 57 & 20 & 21 & 0 & 0 & 0 & 0 & 0 & 0 & 21 \\
\hline Manglares de tierra 2 & 86 & 29 & 21 & 0 & 0 & 0 & 0 & 0 & 0 & 3 \\
\hline \multicolumn{11}{|c|}{ Cayos de la Leña } \\
\hline Cayos de la Leña 1 & 64 & 15 & 23 & 0 & 0 & 0 & 0 & 0 & 0 & 8 \\
\hline Cayos de la Leña 2 & 100 & 23 & 78 & 0 & 0 & 0 & 0 & 0 & 0 & 0 \\
\hline Cayos de la Leña 6 & 0 & 0 & 0 & 0 & 0 & 0 & 100 & 46 & 26 & 0 \\
\hline Cayos de la Leña 4 & 68 & 24 & 25 & 0 & 0 & 0 & 0 & 0 & 0 & 4 \\
\hline Cayos de la Leña 10 & 77 & 18 & 75 & 49 & 22 & 27 & 82 & 41 & 80 & 17 \\
\hline \multicolumn{11}{|c|}{ Palma Sola-Carabelita } \\
\hline Palma Sola & 27 & 17 & 13 & 0 & 0 & 0 & 0 & 0 & 0 & 11 \\
\hline Carabelita & 40 & 21 & 17 & 10 & 22 & 18 & 0 & 0 & 0 & 15 \\
\hline \multicolumn{11}{|c|}{ Punta Plumaje-Melones } \\
\hline Punta Plumaje & 48 & 21 & 21 & 2 & 3 & 16 & 0 & 0 & 0 & 3 \\
\hline Canal Tasajera & 51 & 21 & 14 & 0 & 0 & 0 & 0 & 0 & 0 & 21 \\
\hline \multicolumn{11}{|c|}{ Bahía de la Fe } \\
\hline Bahia de la Fe & 100 & 37 & 31 & 0 & 0 & 0 & 0 & 0 & 0 & 5 \\
\hline
\end{tabular}

área protegida permitirá que se ejecuten sus funciones ecológicas, entre las que podemos citar: el mantenimiento de la biodiversidad, la producción de fuentes directas e indirectas de alimento, la estabilidad de los sedimentos para proteger la costa de la erosión, y fuente de refugio, crianza y alimentación de diferentes especies animales, entre las que podemos citar las poblaciones de tortugas marinas y manatíes.

Los bosques de mangles constituyen la segunda formación vegetal en exten- sión en la península (Delgado \& Ferro, 2002). Están formados, principalmente, por mangle rojo ( $R$. mangle), mangle prieto (Avicennia germinans), patabán (Laguncularia racemosa) y yana (Conocarpus erectus). Estas especies han sufrido considerables afectaciones por huracanes intensos que han azotado a la península, sobre todo por el efecto de las penetraciones del mar que han incrementado los niveles de salinidad. Durante el estudio se observó una considerable recuperación de esta formación vege- 
tal, quedando solo pequeños parches de mangle rojo dañados en la primera línea, lo cual podría deberse al grado de exposición a los vientos del norte.

La parte sumergida del mangle rojo está formada por raíces largas con valores que oscilan entre 43 y $142 \mathrm{~cm}$, las que sirven de refugio a juveniles de invertebrados y peces de importancia comercial y conservacionista. Los mayores valores de cubrimiento y diversidad de las raíces se encontraron en las estaciones más protegidas de los vientos y el oleaje, fundamentalmente en los canalizos interiores de los Cayos de la Leña y del interior de la península.

Estas raíces se encuentran densamente pobladas y los grupos de organismos más representados son: algas, esponjas, ascidias, anémonas, turf, hidrozoos y cirripedios (Cuadro 3).

El grupo de mayor abundancia fue el de las algas, y de las macroalgas los

Cuadro 3. Porcentaje de cubrimiento de organismos sésiles en el frente de las raíces sumergidas de los manglares

Table 3. Percent cover of sessile organisms at the front of the submerged roots of mangroves

\begin{tabular}{|c|c|c|c|c|c|c|}
\hline \multirow{2}{*}{$\begin{array}{l}\text { Zonas } \\
\text { Manglar }\end{array}$} & \multicolumn{3}{|c|}{ Cayos de la Leña } & \multicolumn{2}{|c|}{ Palma Sola-Carabelita } & \multirow{2}{*}{$\begin{array}{c}\text { Bahía de la Fe } \\
\text { La Fe }\end{array}$} \\
\hline & La Leña 1 & La Leña 4 & La Leña 2 & Palma Sola & Carabelita & \\
\hline Raíz analizada $(\mathrm{cm})$ & 1041 & 1798 & 1385 & 1299 & 1351 & 1341 \\
\hline \% de raíces vacías & 41 & 27 & 28 & 66 & 30 & 70 \\
\hline \multicolumn{7}{|c|}{ Cubrimiento por organismos (\%) } \\
\hline Esponjas & 0 & 8 & 12 & 0 & 0 & 0 \\
\hline Algas & 47 & 49 & 35 & 23 & 64 & 24 \\
\hline Turf & 3 & 2 & 6 & 7 & & \\
\hline Ascidias & 7 & 8 & 7 & 0 & 0 & 0 \\
\hline Corales & 0 & 0 & 0 & 0 & 0 & 0 \\
\hline Anémonas & 2 & 4 & 3 & 0 & 0 & 0 \\
\hline Hidrozoos & 0 & 1 & 1 & 0 & 2 & 0 \\
\hline Cirripedios & 0 & 1 & 0 & 0 & 0 & 6 \\
\hline
\end{tabular}

géneros Ceramium sp. y Bostrychia sp. y las especies Amphiroa fragilissima y Caulerpa verticillata fueron las más comunes, las cuales coinciden con lo expuesto por González-Alonso (2007) en otras zonas de Cuba. En el grupo de las esponjas, la más abundante fue Tedania ignis, y de las ascidias, Ecteinascidia turbinata.

Se observaron 53 especies de peces pertenecientes a 18 familias (Cuadro
4). Las especies más abundantes fueron: Jenkinsia lamprotaenia, Eucinostomus gula/havana, Eucinostomus jonesii/ melanopterus, Haemulon sciurus, Lutjanus griseus y Lutjanus apodus. La importancia natural de este ecosistema como sitio de cría de peces comerciales se evidenció con la presencia de especies de pargos en estado juvenil como: Lutjanus jocu, Lutjanus cyanopterus, Lutjanus analis, Lutjanus synagris y Ocyurus chrysurus. 
Cuadro 4. Lista de especies de peces registrados en los manglares estudiados

Tabla 4. Fish species recorded in studied mangroves

\begin{tabular}{lll}
\hline Familia Clupeidae & Eucinostomus gula & Stegastes planifrons \\
Harengula humeralis & Eucinostomus havana & Stegastes variabilis \\
Jenkinsia lamprotaenia & Eucinostomus jonesii & Familia Labridae \\
Familia Belonidae & Eucinostomus melanopterus & Halichoeres bivittatus \\
Strongylura notata & Gerres cinereus & Halichoeres maculipinna \\
Tylosurus crocodilus crocodilus & Familia Haemulidae & Halichoeres radiatus \\
Familia Poeciliidae & Anisotremus virginicus & Thalassoma bifasciatum \\
Gambusia rhizophorae & Haemulon aurolineatum & Familia Scaridae \\
Familia Holocentridae & Haemulon flavolineatum & Scarus guacamaia \\
Sargocentron vexillarium & Haemulon parra & Scarus iserti \\
Familia Scorpaenidae & Haemulon plumieri & Scarus taeniopterus \\
Pterois volitans & Haemulon sciurus & Sparisoma aurofrenatum \\
Familia Carangidae & Archosargus rhomboidalis & Sparisoma rubripinne \\
Caranx latus & Familia Mullidae & Sparisoma viride \\
Caranx ruber & Mulloidichthys martinicus & Hypleurochilus sp \\
Familia Lutjanidae & Familia Chaetodontidae & Familia Acanthuridae \\
Lutjanus analis & Chaetodon capistratus & Acanthurus bahianus \\
Lutjanus apodus & Familia Kyphosidae & Acanthurus chirurgus \\
Lutjanus cyanopterus & Kyphosus incisor & Acanthurus coeruleus \\
Lutjanus griseus & Kyphosus sectatrix & Familia Sphyraenidae \\
Lutjanus jocu & Familia Pomacentridae & Sphyraena barracuda \\
Lutjanus synagris & Abudefduf saxatilis & Familia Balistidae \\
Ocyurus chrysurus & Stegastes leucostictus & Canthidermis sufflamen \\
Familia Gerreidae & Stegastes partitus & \\
\hline & & \\
\hline
\end{tabular}

Se observó al pez loro Scarus guacamaia, en su estado adulto, en las raíces del manglar. Esta especie es rara en los ecosistemas marinos de Cuba y está declarada por la UICN en peligro. También se confirmó por primera vez la presencia de la especie exótica pez león (Pterois volitans/miles) en los ecosistemas de pastos marinos (4 individuos) y manglares (5 individuos), con una abundancia baja y con una talla máxima encontrada de $25 \mathrm{~cm}$ (2 individuos).

Durante los recorridos para el estudio de los manatíes no se observó ningún animal. Sin embargo, en intercambios sostenidos con los pescadores, estos mencionaron que las zonas con frecuencia de avistamientos eran: los Cayos de la Leña, Cayo Triste, la Laguna de Bolondrón y Bahía de la Fe.

Basados en los reportes anecdóticos y en las condiciones observadas en el área, se determinaron dos subzonas 
núcleos para desarrollar el programa de monitoreo de manatí: una primera subzona que se extiende desde Cayo Triste hasta los Cayos de la Leña, e incluye la Laguna de Bolondrón. Una segunda subzona al este de la región que comprende desde la Ensenada de la Fe hasta Punta Colorada (Fig. 3). La primera representa un área potencial de alimentación por la abundancia de pastos marinos y la disponibilidad de refugios naturales para la protección de los animales (cayos y diversidad de canales de profundidades variables). Por otra parte, la segunda zona propuesta fue mencionada como la de mayor incidencia de la especie y resulta importante por la accesibilidad a fuentes de agua dulce, además de la cobertura de pastos marinos. En este lugar se encuentra el poblado de pescadores deportivos de $\mathrm{La} \mathrm{Fe}$, por lo que resulta primordial monitorear los posibles impactos de esta comunidad pesquera en los grupos de manatíes del área.
Durante las observaciones realizadas en el período de muestreo se registraron 55 especies de aves acuáticas y marinas (Cuadro 5). La representación de órdenes y familias fue de 14 y 24 , que representan un $66.6 \%$ y un $38.1 \%$, respectivamente, del total del territorio cubano y un $73.7 \%$ y un $53.3 \%$ para la Península de Guanahacabibes. Las zonas más importantes identificadas para las aves marinas y acuáticas fueron Cayo Triste, Cayos de la Leña, Punta Plumaje y Ensenada de la Fe, en estas se encontraron condiciones favorables de hábitats (alimento, refugio y nidificación) (Fig. 4).

Los órdenes mejor representados fueron: Ciconiformes, Charadriiformes y Passeriformes; destacando las familias Ardeidae, Laridae y Parulidae, respectivamente. Según los criterios de permanencia descritos por Llanes et al. (2002), estuvieron más representadas las especies residentes permanentes con 20 especies que representaron

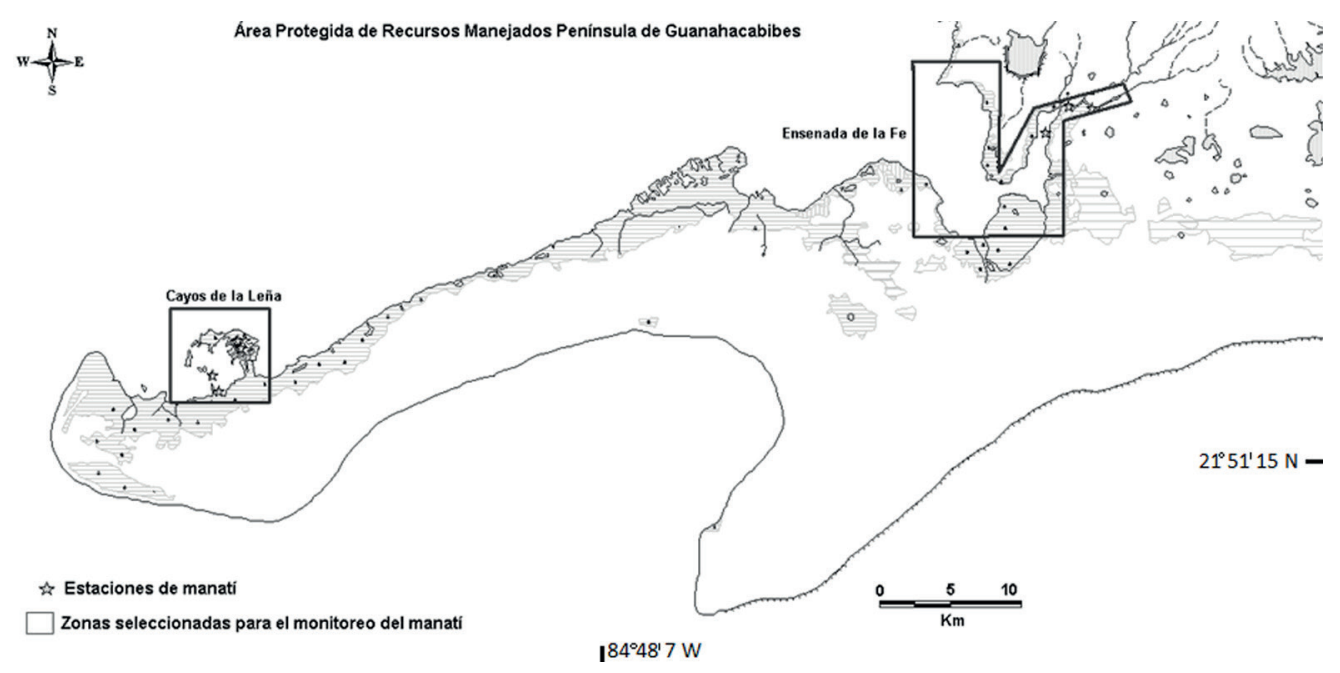

Fig. 3. Zonas importantes para las poblaciones de manatíes en la región

Fig. 3. Important zones for manatee populations 
Cuadro 5. Lista de especies de aves acuáticas y marinas reportadas en la zona de estudio Table 5. Waterfowl and seabird species recorded in the studied area

\begin{tabular}{lll}
\hline Familia Podicipedidae & Accipiter striatus & Familia Columbidae \\
Podilymbus podiceps & Buteogallus gundlachii & Columbina passerina \\
Tachybaptus dominicus & Rostrhamus sociabilis & Familia Cuculidae \\
Familia Anhingidae & Familia Falconidae & Saurothera merlini \\
Anhinga anhinga & Falco sparverius & Familia Alcedinidae \\
Pelecanus occidentalis & Familia Rallidae & Ceryle alcyon \\
Familia Phalacrocoracidae & Fulica americana & Familia Apodidae \\
Phalacrocorax auritus & Familia Scolopacidae & Tachornis phoenicobia \\
Familia Fregatidae & Charadrius wilsonia & Familia Picidae \\
Fregata magnificens & Calidris mauri & Melanerpes superciliaris \\
Familia Ardeidae & Catoptrophorus semipalmatus & Xiphidiopicus percussus \\
Ardea alba & Actitits macularius & Familia Turdidae \\
Ardea herodias & Calidris pusilla & Turdus plumbeus \\
Bubulcus ibis & Familia Recurvirostridae & Familia Parulidae \\
Butorides virescens & Himantopus mexicanus & Mniotilta varia \\
Egretta caerulea & Familia Laridae & Setophaga ruticilla \\
Egretta rufescens & Larus atricilla & Dendroica palmarum \\
Egretta thula & Larus marinus & Seiurus noveboracensis \\
Egretta tricolor & Sterna anaethetus & Geothlypis trichas \\
Familia Threskiornithidae & Sterna antillarum & Familia Cardinalidae \\
Eudocimus albus & Sterna caspia & Passerina cyanea \\
Plegadis falcinellus & Sterna dougallii & Familia Tyrannidae \\
Familia Anatidae & Sterna fuscata & Contopus caribaeus \\
Mergus serrator & Sterna hirundo & Tyrannus caudifasciatus \\
Oxyura jamaicensis & Sterna maxima & Familia Cuculidae \\
Familia Accipitridae & Sterna sandvicensis & Crotophaga ani \\
\hline & & \\
& &
\end{tabular}

un $36.3 \%$ del total registrado, seguidas por las bimodales con 17 (31\%) (que son especies que tienen poblaciones que permanecen todo el tiempo en territorio cubano y otras que migran en determinado período del año), luego, las residentes de invierno con 12 que significaron un $21.7 \%$ y en menor medida, como se esperaba durante este período, las residentes de verano (6 para un 11\%).

Debemos destacar que las observaciones se efectuaron en un período de residencia invernal, pero el número de especies que visitan la península durante su travesía de norte a sur en época migratoria es grande, por lo que la relación de aves que utilizan este litoral debe ser mucho mayor. Se debe destacar también que en la etapa reproductiva cuando las residentes incrementan la vocalización muchos más individuos se pueden hacer notar. 


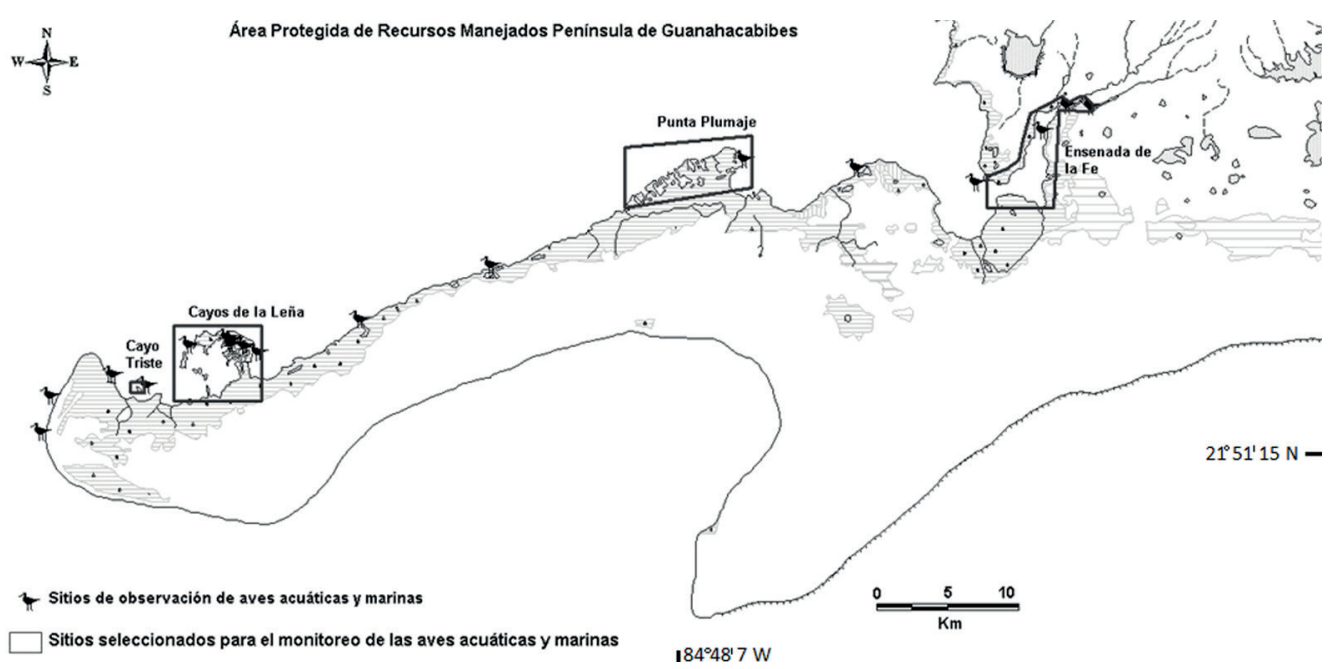

Fig. 4. Sitios importantes para la alimentación, refugio y nidificación de aves marinas y acuáticas Fig. 4. Important bird feeding, refuge, and nesting sites

La totalidad de las aves fueron detectadas sobre el mar, en las áreas de manglar y en la laguna interior. Varias especies fueron comunes, pero se destacaron con altas cifras de abundancia: Larus atricilla, Phalacrocorax auritus y Fregata magnificens, registrándose de esta última una colonia de nidificación en Cayo Triste (Zona Cayos de la Leña) con aproximadamente 300 individuos.

Cayo Triste está formado por pequeños parches de $R$. mangle que conforman un pequeño cayo en el medio del mar, de una extensión estimada de dos kilómetros. En estos, se observó una gran cantidad de nidos construidos, juveniles y parejas en cortejo, lo que evidencia que se encontraban finalizando un ciclo de reproducción y comenzando otro. Los nidos se encontraron en mangles de gran altura, a unos 4 o $5 \mathrm{~m}$.

Según los pescadores, la colonia se ha mantenido por años en este sitio, utilizando los mismos árboles para la nidificación, siendo la mayor actividad reproductiva durante el mes de mayo. La colonia ha sido afectada en otras ocasiones por los daños ocasionados a los manglares por los huracanes frecuentes en la región, sin embargo, se ha mantenido el sitio en la zona. Esta área es utilizada por otras especies como son los casos de las corúas y la garza tricolor. El sitio de nidificación en cuestión es altamente sensible por sus características y por su importancia; en caso de ser utilizado para el ecoturismo, en la modalidad de observación de avifauna, se deben tener en cuenta acciones de conservación y manejo establecidas por el APRM.

F. magnificens (rabihorcados) es un residente común de cayos y costas de Cuba. Existen ocho sitios de nidificación en el país, pero hasta el momento solo cuatro tienen estimados poblacionales. El sitio no se había documentado hasta este momento, siendo el segundo sitio de nidificación 
más importante en el país, a continuación de un sitio en Cayo Coco (Ciego de Ávila) (Bradley \& Norton, 2009). También se reportó por los pescadores de la zona otro sitio de nidificación en los manglares de la zona de Punta Plumaje de las especies Eudocimus albus y Plegadis falcinellus (cocos).

\section{Especies amenazadas y nuevos repor- tes para el APRM}

En el área estudiada se reportaron especies amenazadas según la Unión Internacional para la Conservación de la Naturaleza (UICN). Cinco especies de peces de interés conservacionista, cuatro declaradas como vulnerables (L. analis, $L$. cyanopterus, $L$. griseus, L. synagris y una en peligro crítico ( $S$. guacamaia). Las tortugas marinas Chelonia mydas (tortuga verde), Caretta caretta (caguama), Eretmochelys imbricata (carey), declaradas en peligro, registradas en los pastos marinos (sitios de alimentación), de igual manera $T$. manatus manatus (manatí), con categoría de vulnerable. Se observaron las especies de aves Buteogallus gundlachii, Egretta rufescens y Calidris pusilla, todas en la categoría de Cercano a la amenaza (NT), según la IUCN (2012).

Se registraron 24 especies nuevas de algas pertenecientes a los filos Rhodophyta (9) y Chlorophyta (11), así como dos especies de Magnoliophyta (Cuadro 6).

Con respecto a la fauna, se identificaron 2 especies de la clase Platyhelminthes, 2 de la clase Cnidaria, 4 de la clase Porífera, 3 de la clase Equinodermata, 2 de la clase Arthropoda y 2 de la clase Annelida, para un total

Cuadro 6. Nuevos reportes de algas y pastos marinos para la costa norte del APRM Península de Guanahacabibes (Ecorregión 6)

Table 6. New reports of algae and phanerogam species for the Northern Coast of the Guanahacabibes Peninsula APRM (Eco-Region 6)

\begin{tabular}{lclc}
\hline Flora marina & Ecorregión 6 & Flora marina & Ecorregión 6 \\
\hline Filo Rhodophyta & & Avrainvillea nigricansf.floridana & $\mathrm{x}$ \\
Acanthophora spicifera & $\mathrm{x}$ & Caulerpa ashmeadii Harvey & $\mathrm{x}$ \\
Bryotamnion seafortii & $\mathrm{x}$ & Caulerpa paspaloides & $\mathrm{x}$ \\
Chondria atropurpurea & $\mathrm{x}$ & Caulerpa prolifera & $\mathrm{x}$ \\
Chondria polyrhiza & $\mathrm{x}$ & Cladocephalus luteofuscus & $\mathrm{x}$ \\
Dasya baillouviana & $\mathrm{x}$ & Cladophora prolifera & $\mathrm{x}$ \\
Dasya harveyi Ashmead & $\mathrm{x}$ & Halimeda simulans Howe & $\mathrm{x}$ \\
Gelidiopsis intricata & $\mathrm{x}$ & Halimeda tuna & $\mathrm{x}$ \\
Hypoglossum hypoglossoides & $\mathrm{x}$ & Valonia aegaropila & $\mathrm{x}$ \\
Wrightiella tumanowiczii & $\mathrm{x}$ & Filo Magnoliophyta & \\
Filo Chlorophyta & & Halodule wrigtii & $\mathrm{x}$ \\
Avrainvillea nigricans & $\mathrm{x}$ & Halophila engelmanni & $\mathrm{x}$ \\
Avrainvillea nigricans $f$. spongiosa & $\mathrm{x}$ & & \\
\hline
\end{tabular}


de 15 especies, 13 de ellas reportadas para la ecorregión 6 a la que pertenece la costa norte del APRM y dos registros para Cuba (Cuadro 7). Dos nuevos registros de aves para la Península de Guanahacabibes: Rostrhamus sociabilis (Gavilán Caracolero), que en la provincia tenía su área de distribución restringida al Municipio Sandino, pero no al territorio en estudio, y Calidris mauri (Zarapico Chico), residente de invierno en nuestro país.

En la zona de estudio se encontraron sitios prioritarios para la conservación $\mathrm{y}$ manejo de hábitats $\mathrm{y}$ especies como: zonas de cría de especies de invertebrados y peces comerciales en los manglares (Cayos de la Leña y canalizos interiores de la península); ecosistemas de pastos marinos desde las raíces de los mangles hasta la isobata de $3 \mathrm{~m}$ (sitios de alimentación de tortugas marinas y manatíes, y refugio de especies); sitios de nidificación de aves marinas (CayoTriste y Punta Plumaje) y hábitats importantes para los manatíes (Bahía de la Fe y Cayos de la Leña).

Los principales problemas detectados en el área fueron: la presencia del pez león en los ecosistemas de manglar y pastos marinos; la degradación o alteración de hábitats y disturbios a las comunidades de aves durante la época de nidificación por intromisión de personal; la caza furtiva del manatí y afectaciones por

Cuadro 7. Nuevos reportes de la fauna marina para la costa norte del APRM Península de Guanahacabibes (Ecorregión 6) y para Cuba

Table 7. New reports of fauna species for the Northern Coast of the Guanahacabibes Peninsula APRM (Eco-Region 6) and for Cuba

\begin{tabular}{|c|c|c|c|c|}
\hline Especies & Ecorregión 6 & Cuba & Especies & Ecorregión 6 \\
\hline Filo Platyhelminthes & & & Filo Echinodermata & \\
\hline Familia Pseudocerotidae & & & Familia Holothuriidae & \\
\hline Pseudoceros pardalis & $\mathrm{x}$ & $\mathrm{x}$ & Holothuria (Halodeima) grisea & $\mathrm{x}$ \\
\hline Pseudoceros splendidus & $\mathrm{x}$ & $\mathrm{x}$ & Actinopyga agassizi & $\mathrm{x}$ \\
\hline Filo Cnidaria & & & Familia Toxopneustidae & \\
\hline Familia Plexauridae & & & Lytechinus variegatus carolinus & $\mathrm{x}$ \\
\hline Muriceopsis flavida & $\mathrm{x}$ & & Filo Arthropoda & \\
\hline Plexaurella nutans & $\mathrm{x}$ & & Familia Stenopodidae & \\
\hline Filo Porifera & & & Stenopus hispidus & $\mathrm{x}$ \\
\hline Familia Chondrillidae & & & Familia Diogenidae & \\
\hline Chondrilla aff. nucula & $\mathrm{x}$ & & Paguristes puncticeps & $\mathrm{x}$ \\
\hline Familia Pachastrellidae & & & Filo Annelida & \\
\hline Calyx podatypa & $\mathrm{x}$ & & Familia Terebellidae & \\
\hline Familia Plakinidae & & & Eupolymnia crassiccornis & $\mathrm{x}$ \\
\hline Plakortis angulospiculatus & $\mathrm{x}$ & & Familia Amphinomidae & \\
\hline $\begin{array}{l}\text { Familia Aplysinidae } \\
\text { Verongula rigida }\end{array}$ & $\mathrm{x}$ & & Hermodice carunculata & $\mathrm{x}$ \\
\hline
\end{tabular}


contaminación en la Bahía de la Fe producto de los ríos Verde y Guadiana donde se vierten aguas residuales y desechos sólidos.

De forma general, los recursos marinos importantes se encuentran dentro de los límites del APRM. Sin embargo, se propone ampliar los límites por la costa norte de la península hasta la isobata de $5 \mathrm{~m}$ para incluir la totalidad de los pastos marinos, además de ampliar los límites a partir de la Ensenada de la Fe hasta Punta Colorada, con el objetivo de proteger las poblaciones de manatíes del área.

La ejecución de este estudio permitió establecer la línea base de la zona, conocer el estado de salud de los ecosistemas costeros y las problemáticas existentes tanto de origen natural como antrópico. Los resultados obtenidos permitirán establecer los programas de monitoreo de la zona, así como trazar estrategias de protección y manejo sostenible, relacionadas con la conservación de los ecosistemas y especies claves al norte del Área Protegida de Recursos Manejados Península de Guanahacabibes.

\section{BIBLIOGRAFÍA}

Azanza, J., Ibarra, M., Espinosa, G., Díaz, R. \& González-Sansón, G. (2003). Conducta de la anidación de tortuga verde (Chelonia mydas) en las playas de Antonio y Caleta de los Piojos en la Península de Guanahacabibes. Pinar del Río, Cuba. Rev. Invest. Mar., 24(3), 231-240. Azanza, J., Ibarra, M. E. ${ }^{\dagger}$ Espinosa, G., Bretos, F., Cobián, D., Angulo, J., González, G., Márquez, L. \& Hernández,
N. (2012, octubre). 14 años de trabajo del "Proyecto universitario para el estudio y conservación de tortugas marinas en Cuba". Ponencia presentada en el XI Congreso de Ciencias del MarMARCUBA, Ciudad de La Habana, Cuba.

Bradley, P. E. \& Norton, R. L. (2009). An inventory of breeding seabirds of the Caribbean. Florida, EE.UU.: University Press of Florida.

Brock, V. J. (1954). A preliminary report on a method of estimating reef fishes populations. J. Wildl. Manage., 18(3), 297-308.

Caballero, H., González-Ferrer, S., Cobián, D., Álvarez, S. \& Alcolado, P. M. (2007). Evaluación AGRRA del bentos en diez sitios de buceo de "María la Gorda", Bahía Corrientes, Cuba. Rev. Invest. Mar., 28(2), 131-138.

CARICOMP. (2001). CARICOMP Methods Manual - Level I. CARICOMP Management Center, University of the West Indies, Mona, Kingston, Jamaica and Florida. Institute of Oceanography, University of South Florida, St. Petersburg Florida, EE.UU. Mona, Kingston, Jamaica.

Claro, R. \& Cantelar-Ramos, K. (2003). Rapid assessment of the coral communities of María La Gorda, southeast Ensenada de Corrientes, Cuba (Part 2: reef fishes). Atoll. Res. Bull., 496, 278-293.

Cobián, D., González, R. \& Berovides, V. (2008). Densidad de la iguana cubana (Cyclura nubila nubila) en la zona de los farallones del Parque Nacional Guanahacabibes, Pinar del Río, Cuba. Mesoamericana, 12(1), 6-14.

Cobián, D. \& Chevalier Monteagudo, P. P. (2009). Evaluación de las asociaciones de peces de los arrecifes coralinos del 
Centro Internacional de Buceo María la Gorda, Parque Nacional Guanahacabibes, Cuba. Rev. Mar. Cos., 1, 111-125.

Cobián, D., Claro, R., Chevalier Monteagudo, P., Perera, S. \& Caballero, H. (2011). Estructura de las asociaciones de peces en los arrecifes coralinos del Parque Nacional Guanahacabibes, Cuba. Rev. Mar. Cos., 3, 153-169.

Delgado, F. \& Ferro, J. (2002). Nuevas variantes estructurales en la vegetación de los manglares de la Reserva de Biosfera Península de Guanahacabibes, Cuba. En L. M. Carrera, \& J. M. Guzmán (Eds.), Ecosistemas de Manglar en el Archipiélago Cubano (pp. 302-309). La Habana, Cuba: Editorial Academia.

Espinosa, J., Ortea, J., Caballer, M. \& Moro, L. (2005). Moluscos Marinos de la Península de Guanahacabibes, Pinar del Río, Cuba, con la descripción de nuevos taxones. Avicennia, 18, 1-84.

Espinosa, J., Ortea, J., Fernández-Garcés, R. \& Moro, L. (2007). Adiciones a la fauna de moluscos marinos de la Península de Guanahacabibes (I), con la descripción de nuevas especies. Avicennia, 19, 63-88.

González-Alonso, H. (2007). Biota Marina. En H. González-Alonso (Ed.), Biodiversidad de Cuba (pp. 84-85). La Habana, Cuba: Ediciones Polymita.

González-Ferrer, S., Caballero, H., Alcolado, P. M., Jiménez, A., Martín, F. \& Cobián, D. (2007). Diversidad de corales pétreos en once sitios de buceo recreativo de "María la Gorda", Cuba. Rev. Inv. Mar., 28(2), 121-130.

IUCN. (2012). IUCN Red List of Threatened Species. Version 2012. Recuperado en febrero 08, 2013, disponible en http://www.iucnredlist.org.
Littler, D. S. \& Littler, M. M. (2000). Caribbean reef plants. An identification guide to the reef plants of the $\mathrm{Ca}$ ribbean, Bahamas, Florida and Gulf of México. Washington D. C., EE.UU.: OffShore Grafics, Inc.

Llanes, A., González, H., Sánchez, B. \& Pérez, E. (2002). Lista de las Aves Registradas para Cuba. En H. GonzálezAlonso (Ed.), Aves de Cuba (pp. 147155). Vaasa, Finlandia: UPC Print.

Martínez, D. B., Cabrera, R. \& PinaAmargos, F. (2011). Spatial and seasonal variability of Thalassia testudinum in Nuevitas Bay, Cuba. Rev. Mar. Cos., 1, 9-27.

McKenzie, L. J., Campbell, S. J. \& Roder, C. A. (2003). Seagrass-Watch: Manual for Mapping \& Monitoring Seagrass Resources by Community (citizen) volunteers. Townsville, Australia: QFS, NFC, Cairns.

Metaxas, A., Scheibling, R. E. \& Young, C. M. (2002). Estimating fertilization success in marine benthic invertebrates: a case study with the tropical sea star Oreaster reticulatus. Mar. Ecol. Prog. Ser., 226, 87-101.

Myers, R. \& Ottensmeyer, A. (2005). Marine conservation Biology, the science of maintaining the sea's biodiversity. In E. Norse \& L. Crowder (Eds.), Extinction risk in marine specie (pp. 58-79). Washington, EE.UU.: Marine Conservation Biology Institute.

Perera, S. (2010). Influencia de la exposición a vientos generadores de oleaje sobre comunidades de corales pétreos del Parque Nacional Guanahacabibes, Cuba. Tesis de maestría no publicada, Universidad de La Habana, Cuba.

Ramsar. (2013). Manual de la Convención de Ramsar: Guía a la Convención sobre los Humedales (Ramsar, Irán, 
1971). Gland, Suiza: Secretaría de la Convención de Ramsar

Rogers, C. S., Garrison, G., Grober, R., Hillis, Z. M. \& Franke, M. A. (1994). Coral reef monitoring manual for the Caribbean and Western Atlantic. Virgin Islands National Park, EE.UU.: Sin editorial.

Short, F. T. \& Wyllie-Echeverría, S. (1996). Natural and human-induced disturbance of seagrasses. Environ. Conserv., 23(1), 17-27. Smethurst, D. \& Nietschmann, B. (1999). The distribution of manatees (Trichechus manatus manatus) in the coastal waterways of Tortuguero, Costa Rica. Biol. Conserv., 89, 267-274.
Taylor, W. R. (1960). Marine algae of the Eastern Tropical and Subtropical coasts of the Americas. Michigan, EE.UU.: The University of Michigan Press.

Valdivia, A., de la Guardia, E., Armenteros, M., González, P., Suárez, A. M., Aguilar, C. \& González-Sansón, G. (2004). Inventario de los componentes más comunes de la flora y la fauna de algunos arrecifes coralinos de la Península de Guanahacabibes, Pinar del Río, Cuba. Rev. Inv. Mar., 25(2), 113-121.

Vázquez, A. A. \& Cobián, D. (2008). Moluscos fluviales de la Península de Guanahacabibes. Revista ECOVIDA, 1(1), 1-7. 